\title{
Potentials of representative heirloom vegetables on Shonai region of Yamagata, Japan
}

\author{
Takeshi Nagai ${ }^{1,2,3 *}$, Takumi Nagata ${ }^{4,5}$, Yasuhiro Tanoue ${ }^{6}$, Norihisa Kai ${ }^{7}$, Nobutaka Suzuki ${ }^{8}$ \\ ${ }^{1}$ Graduate School of Agricultural Sciences, Yamagata University, Yamagata 9978555, Japan, ${ }^{2}$ The United Graduate School of Agricultural \\ Sciences, Iwate University, Iwate 0208550, Japan, ${ }^{3}$ Graduate School, Prince of Songkla University, Songkhla 90112, Thailand, ${ }^{4}$ Yamagata \\ University, Yamagata 9978555, Japan, ${ }^{5}$ Yataro Group, Shizuoka 4350046, Japan, ${ }^{6}$ National Fisheries University, Yamaguchi 7596595, Japan, \\ ${ }^{7}$ Oita University, Oita 8701192, Japan, ${ }^{8}$ Nagoya Research Institute, Aichi 4701131, Japan
}

\section{A B S TR A C T}

\begin{abstract}
The aim of this work was to elucidate the proximate composition, functional components, and functional properties of representative heirloom vegetables on Shonai region of Yamagata, Japan. Turnip roots such as Fujisawakabu and Tomoefuki contained a lot of proteins and carbohydrates among these vegetables tested. Many vegetables showed about 2-3 times as many vitamin $C$ and $\beta$-carotene values as corresponding commercially available vegetables. Overall, water and methanol extracts prepared from these vegetables possessed remarkably high antioxidative activities except for Makomodake. Radical scavenging through different mechanisms and hyaluronidase inhibitory activities varied markedly among these vegetables. Particularly, all vegetables exhibited outstanding ACE inhibitory activities about 45.1-95.8\%. These findings demonstrated that heirloom vegetables used in this study served as good sources of vitamins, phenolics, and antioxidants compared to corresponding commercially available vegetables. Positively eating of these vegetables can probably contribute to health promotion to prevent life style-related diseases such as cancer, hypertension, and inflammation. Furthermore, it also may have potentials for preservation of species and for promotion of sustainable cultivation of heirloom vegetables.
\end{abstract}

Keywords: Functional property; Heirloom vegetable; Nutrition; Proximate composition

\section{INTRODUCTION}

Vegetables plays important roles as not only resources for supplying micronutrients such as vitamins and minerals that are essential for normal nutrition and metabolisms but also as low-caloric and low-fatty foods. In addition, many kinds of functional ingredients contain in it. Phenolics have multiple biological functions such as antioxidant and antibacterial properties (Fawole et al., 2012) and synergistic effects and protective properties against life style-related diseases such as arteriosclerosis, arthritis, brain dysfunction, cardiovascular diseases, cancer, hypertension, and inflammation (Verma et al., 2018). Reactive oxygen species as superoxide anion radicals, hydrogen peroxide, and hydroxyl radicals, which cause oxidative damage of human body, are factors of onset of many diseases containing cancer. Therefore, consumption of vegetables, which are rich in fibers, minerals, phenolics, and vitamins, help to prevent oxidative stress and to reduce incidence of these diseases (Fidrianny et al., 2018). Moreover, it is reported that intake of antioxidants such as phenolics and vitamins retard ageing (Ross and Kasum, 2002).

Heirloom vegetables are crops that seeds are maintained and conservation of species is continued by growers in limited areas over generations. It is considered that these species have values as cultural properties because these are genetic resources for breeding materials and are in close relations with traditional eating habits in a region. However, traditional varieties rapidly disappear worldwide, therefore, it has been discussing the way of conservation of varieties (Tomiyoshi and Ueno, 2016). Recently, there is a nationwide trend to revalue existence and benefits of heirloom vegetables. Representative heirloom vegetables in Japan are as follows: Shishigatani pumpkin, Kujonegi, and Fushimi pepper in Kyoto (JA-Kyoto), Aizumaru eggplant, Arakudakukitachi, and Tachikawa burdock in Aizu region of Fukushima (Association to protect traditional vegetables in Aizu; Mizuno and Sumino, 2008), and Nakajimana, Futatsukakarashina, and Kaga Glycine soja in Kanazawa

\footnotetext{
*Corresponding author:

Takeshi Nagai,Graduate School of Agricultural Sciences, Yamagata University, Yamagata 9978555, Japan.

E-mail: nagatakenagatake@yahoo.co.jp, tnagai@tds1.tr.yamagata-u.ac.jp

Received: 20 December 2018 ; $\quad$ Accepted: 12 February 2019
} 
region of Ishikawa (Kanazawa City Agricultural Products Branding Association). Particularly, there are many types of heirloom vegetables in Yamagata, Japan (179 varieties in 2018). Among them, 87 varieties of vegetables are cultivated in Shonai region of Yamagata, Japan (Tsuruoka Creative City of Gastronomy Promotion Committee, 2018). However, few studies have explored even proximate composition and nutritional properties of these vegetables. It is expected that many vegetables are more nutritious than commonly available vegetables, as these has not undergo selective breeding aimed at good appearance and convenience for broad area distribution. Consumers nowadays tend to require foods with high nutritional values and health functionalities. This work was performed to evaluate proximate composition and physicochemical and nutritional properties of main heirloom vegetables on Shonai region of Yamagata, Japan, and furthermore to elucidate health functionalities of these vegetables.

\section{MATERIALS AND METHODS}

\section{Materials}

Fourteen fresh heirloom vegetables (Atsumikabu, Chijimina, Fujisawakabu, Hirataakanegi, Karatori, Kirariboshi, Makomodake, Mindennasu, Mosodake, Natsuna, Okitanasu, Tagawakabu, Tomoebuki, and Tonojimakyuri) were purchased from produce stands on Shonai region of Yamagata, Japan (Fig. 1), and were used in the study. These are representative heirloom vegetables on this region. In addition, these are eaten frequently on the region. The information about these genetic materials is summarized in the book (Yamagata Forum for the Indigenous Crops, 2012). All chemicals were of regent grade.

\section{Determination of proximate composition}

Moisture was measured using a Moisture Determination Balance (FD-600; Kett Electric Laboratory, Tokyo, Japan). Crude proteins were determined by the Kjeldahl method using a conversion factor of 6.25. Crude lipids were analyzed by ether extraction. Measurements of crude ashes were used an electric furnace (AMI-II; Nitto Kagaku Co. Ltd., Aichi, Japan). Carbohydrates were calculated by difference. Crude fibers were measured as described by Nakamura et al. (1998). Salts were determined using a digital salinity concentration meter (EB-158P, EISHIN Co., Ltd., Hiroshima, Japan). Calories were calculated using the FAO conversion factors (Isobe et al., 2011).

\section{Physicochemical properties}

Total soluble solids (TSS) were determined using a digital refractometer (PAL-Pâtissier, Atago Co. Ltd., Tokyo, Japan). The $\mathrm{pH}$ values were measured using a digital $\mathrm{pH}$ meter (PHL-40, DKK-TOA Co., Tokyo, Japan). Alkalinity was estimated as described by Miura et al. (2006). Free

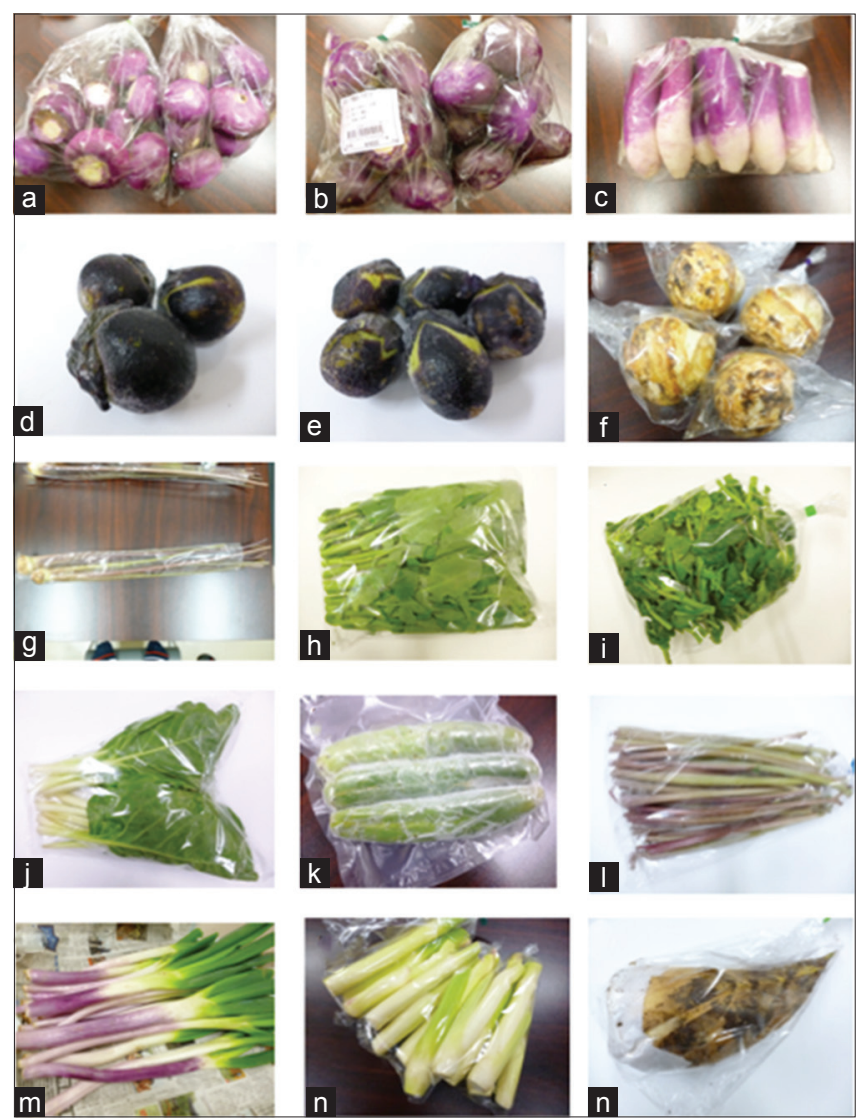

Fig 1. Heirloom vegetables on Shonai region of Yamagata, Japan used in the study. (a) Atsumikabu, (b) Tagawakabu, (c) Fujisawakabu, (d) Okitanasu, (e) Mindensasu, (f) Karatori (tubers), (g) Karatori (stems), (h) Kirariboshi, (i) Chijimina, (j) Natsuna, (k) Tonojimakyuri, (l) Tomoefuki, (m) Hirataakanegi, (n) Makomodake, (o) Mosodake.

amino acid contents were determined by the TNBS method (Sugawara and Soejima, 1996) using L-leucine as standard. Vitamins B1 and B2 were measured by the $p$-aminoacetophenone method and by the lumiflavin fluorescence method, respectively (Nakamura et al., 1998). Vitamin $\mathrm{C}$ was determined by $\alpha, \alpha$ '-dipyridyl method (The Vitamin Society of Japan, 1990). $\beta$-Carotene, lycopene, and chlorophyll $(\mathrm{a}, \mathrm{b})$ were measured using acetone-hexane extraction method (Nagata and Yamashita, 1992). Phenols and flavonoids were determined as described by Slinkard and Singleton (1977) and by Kim et al. (2003), respectively, using quercetin dihydrate as standard.

\section{Functional properties}

Antioxidative activities of extracts from vegetables were evaluated as described by Nagai et al. (2018). Ascorbic acid (AA), tert-butyl-4-hydroxyanisole (BHA), 2,6-di-t-butyl-4methylphenol (BHT), $\alpha$-tocopherol (TP), and trolox (TL) were used as positive controls, and distilled water or $80 \%$ methanol were used as negative one. Superoxide anion radicals, hydroxyl radicals, and DPPH radicals scavenging activities were determined as described by Nagai et al. (2018). Activities [TL equivalents scavenging capacity 
(TESC); $\mathrm{mM} \mathrm{TE} / \mathrm{kg} \mathrm{FW]} \mathrm{were} \mathrm{also} \mathrm{expressed} \mathrm{as} \mathrm{millimoles}$ of TL equivalents per $\mathrm{kg}$ of fresh weight of vegetables. ACE and hyaluronidase inhibitory activities were measured as described by Nagai et al. (2018).

\section{Statistical analysis}

Each assay was repeated 3 times independently and results were reported as means \pm standard deviation (SD).

\section{RESULTS AND DISCUSSION}

\section{Proximate composition}

Proximate composition per $100 \mathrm{~g}$ of vegetables is shown in Table 1. Moisture contents ranged from 90.1-96.0 g except for Karatori tubers and Chijimina. Content in Karatori tubers was similar to eddoe fresh bulbs, but was fairly lower than taro fresh bulbs (Kagawa, 2018). Protein contents were high in Kirariboshi about $3.4 \mathrm{~g}$, followed by Chïimina, Fujisawakabu, and Mosodake, whereas Karatori stems were lowest. Turnip roots showed higher content than commercially available turnip (fresh roots with skin). Lipids were not detected at all or were low about $0.1 \mathrm{~g}$ except for Kirariboshi, Chijimina, and Natsuna. Karatori tubers contained large amount of carbohydrates, suggesting existence of great quantities of starches. Fiber contents were high in leafy vegetables such as Kirariboshi, Chijimina, and Natsuna. Ash contents ranged from 0.4-1.2 g. Salts were not mostly detected in these vegetables. Highest energy was calculated in Karatori tubers about $95.8 \mathrm{kcal}$, which carbohydrate content was highest among these vegetables, followed by in Tomoefuki, Hirataakanegi, Kirariboshi, and Mosodake.

\section{Physicochemical properties}

Vegetables were cut into small pieces, ground in a mortar, and then TSS and $\mathrm{pH}$ at $20^{\circ} \mathrm{C}$ were measured. Highest TSS value was detected in Chijimina, followed by in Hirataakanegi, Atsumikabu, and Okitanasu, whereas Karatori tubers were lowest because of existence of great quantities of starches (Table 1). Correlation between carbohydrate contents and TSS contents was with $\mathrm{R}^{2}=$ 0.648 except for Karatori tubers and Tomoefuki and was with $\mathrm{R}^{2}=0.833$ except for Fujisawakabu. The $\mathrm{pH}$ values ranged from 5.4-6.4. High alkarinity was shown in Chijimina, followed by Makomodake, Kirariboshi, and Fujisawakabu, whereas Tonojimakyuri, Mindennasu, and Okitanasu were low. There were varietal differences on alkarinity among turnip roots and leafy vegetables. Generally, grains, meats, and fish and shellfishes are classified as acid foods, whereas vegetables, fruits, mushrooms, and seaweeds are categorized as alkaline ones. Highest free amino acid contents were shown in Mosodake, followed by in Chijimina, Kirariboshi, and Makomodake, whereas Karatori stems were low.

\section{Functional components}

Kirariboshi showed high vitamin B1 value, followed by Chijimina and Natsuna, whereas turnip roots as Fujisawakabu, Karatori tubers, Tomoefuki, and Makomodake were low (Table 1). Vitamin B2 contents were highest in Chijimina about $0.15 \mathrm{mg}$, followed by in Natsuna, Kirariboshi, and Mindennasu. Karatori (stems and tubers) and Tonojimakyuri were lowest. Chijimina showed more than two times as high content as green pak choi fresh leaves. Tagawakabu possessed high vitamin $C$ about $61.9 \mathrm{mg}$, followed by Fujisawakabu, Kirariboshi, Atsumikabu, and Chijimina, whereas Makomodake and Tomoefuki were lowest. Contents on turnip roots as Atsumikabu were about 2.2-3.3 times as high as commercially available turnip. Okitanasu and Mindennasu showed vitamin $\mathrm{C}$ about 2.5-3.3 times as high as eggplant and Beinasu. Yamaguchi et al. (2012) measured ascorbic acid contents in 12 traditional vegetables (Yamatoyasai) of Nara, Japan. Himotogarashi exhibited high value about $28.0 \mathrm{mg} / 100 \mathrm{~g}$ FW, followed by Murasakitogarashi, Yamatonama, and Chisujimizuna (about 22.4-25.8 mg/100 g FW). Except for Karatori, $\beta$-carotene contents were significantly higher than those on commercially available vegetables. Lycopene was not detected in all vegetables. Chlorophyll a and $\mathrm{b}$ showed high values in leafy vegetables such as Kirariboshi and Natsuna.

Next, vegetables were cut into small pieces, and were homogenized with 2 volumes of distilled water or $80 \%$ methanol. Suspension was centrifuged at $30,000 \mathrm{x} g$ for $30 \mathrm{~min}$ at $4^{\circ} \mathrm{C}$, and supernatants were filtered with glass wool (distilled water extracts: WE; methanol extracts: ME). Phenols and flavonoids contents of these extracts are shown in Table 1. In WEs, Chijimina, Kirariboshi, and Tomoefuki showed high phenols values about 175.9, 147.0, and $137.1 \mathrm{mg}$, respectively, followed by Mosodake and Okitanasu. Fujisawakabu and Makomodake were low. In MEs, Tomoefuki was highest about $361.4 \mathrm{mg}$, followed by Chijimina, Kirariboshi, and Mindennasu, whereas Tonojimakyuri and Atsumikabu were low. These are supported by the findings that butterburs contain a large amount of phenol compounds such as chlorogenic acid, kaempferol, quercetin, fukinolic acid, and fukinone. In WEs, Kirariboshi and Chijimina showed highest flavonoids values about 145.5 and $141.7 \mathrm{mg}$, respectively, followed by Tomoefuki. Hirataakanegi, Makomodake, and Tonojimakyuri. Positive correlation was observed with $\mathrm{R}^{2}=0.753$ between phenols and flavonoids contents in WEs $\left(\mathrm{R}^{2}=0.938\right.$ except for Mosodake). Flavonoids contents accounted for 90.1-99.0\% to phenols of WEs (Kirariboshi, Mindennasu, and Karatori stems and tubers): most of polyphenols was accounted for flavonoids. Meanwhile, its rates were low in Mosodake (13.9\%), Hirataakanegi (20.7\%), Makomodake (30.0\%), and Tonojimakyuri (33.1\%), suggesting existence of large quantities of phenolic components except for 


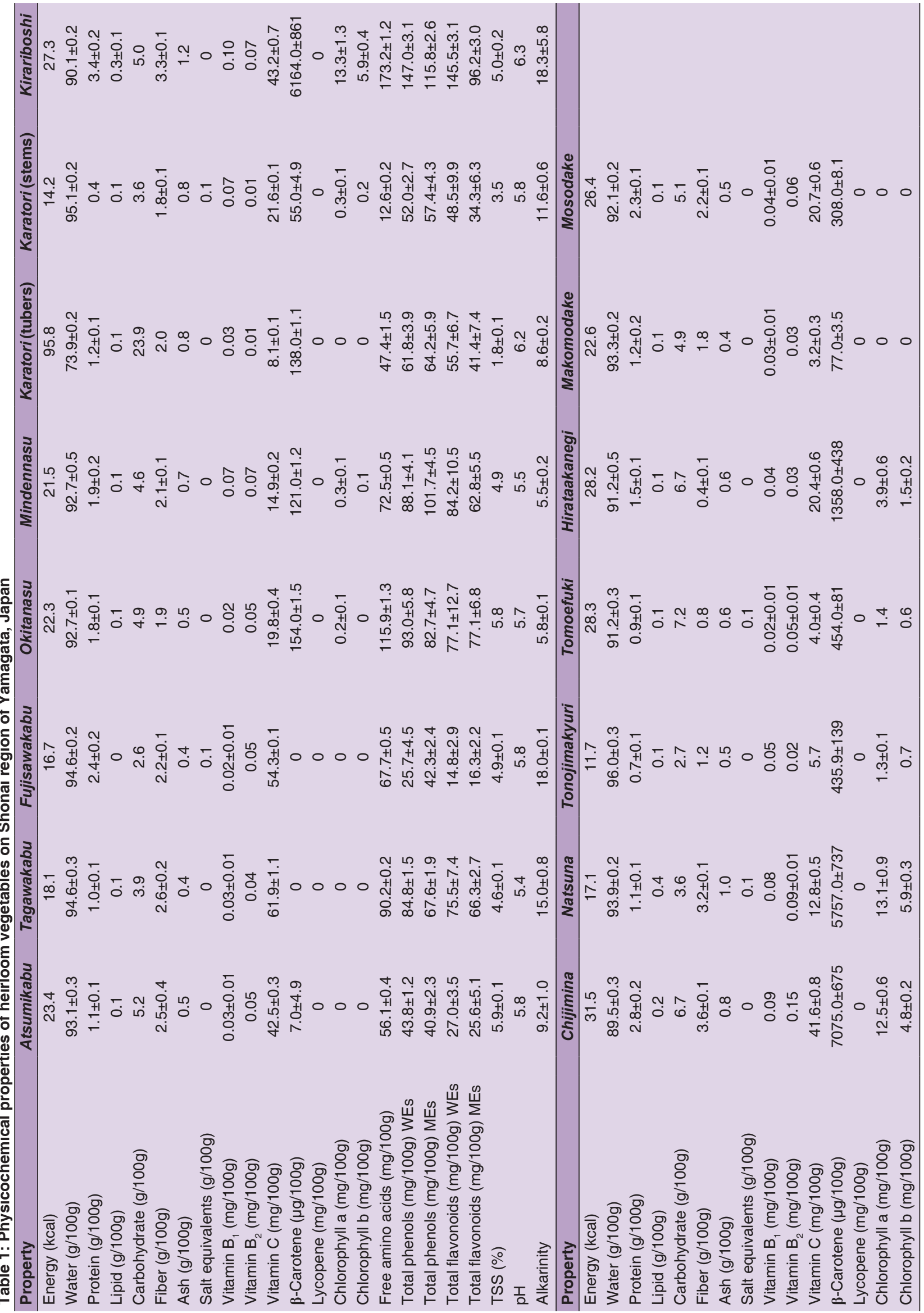




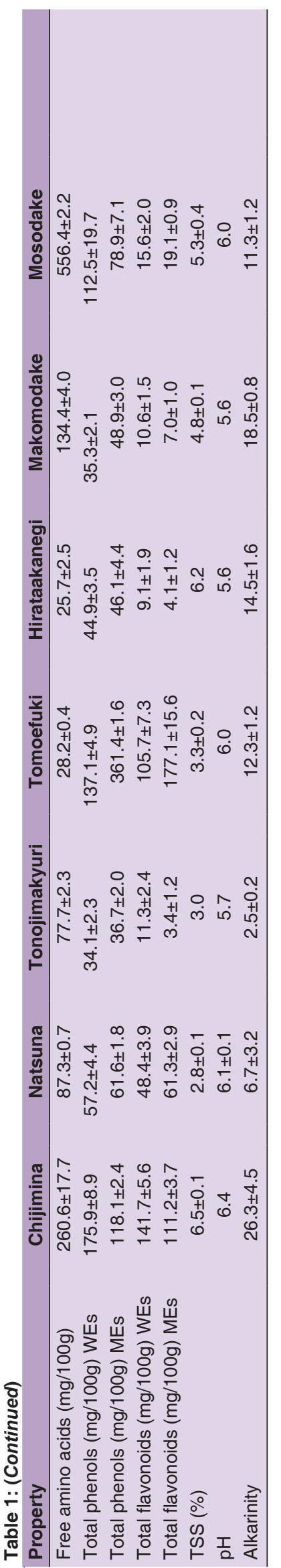

flavonoids. In MEs, Tomoefuki showed highest flavonoid value, followed by Chijimina and Kirariboshi, whereas Tonojimakyuri, Hirataakanegi, and Makomodake were low. Positive correlation with $\mathrm{R}^{2}=0.761$ was observed between phenols and flavonoids contents. The rates of flavonoids to phenols were significantly high in Natsuna, Tagawakabu, Chijimina, and Okitanasu (93.2-99.5\%). Cultivars, preharvest climate conditions, and maturities of vegetables can be defined as crucial factors affecting these functional components biosynthesis and accumulation. Yamaguchi et al. (2012) reported phenol contents in 12 Yamatoyasai vegetables: Yamatonama showed highest value about $5.9 \mathrm{mg}$ gallic acid equivalent (GAE) $/ \mathrm{kg} \mathrm{FW}$, followed by Kaorigobo and Udakingobo (5.4 mg GAE/kg FW), whereas Hanshirokyuri and Yamatosanzyakukyuri were low about 0.8-1.0 mg GAE/kg FW. Itou et al. (2010) investigated polyphenol contents of 12 root vegetables in Ehime, Japan. Yamagobo contained high polyphenols [2.3 g chlorogenic acid equivalent $(\mathrm{CAE}) / \mathrm{kg} \mathrm{FW}$, followed by lotus root (2.1 g CAE $/ \mathrm{kg} \mathrm{FW)} \mathrm{and} \mathrm{Shodaikon} \mathrm{(1.0} \mathrm{g} \mathrm{CAE} / \mathrm{kg} \mathrm{FW).}$ Jersey cudweed (Gogyo) showed significantly high content (8.1 g CAE $/ \mathrm{kg} \mathrm{FW)} \mathrm{compared} \mathrm{to} \mathrm{other} \mathrm{leafy} \mathrm{vegetables}$ such as common henbit and shepherd's purse. Moreover, Kinukawanasu showed moderate value about $3.8 \mathrm{~g}$ CAE $/ \mathrm{kg}$ FW, but loquat hacha was lowest about $0.1 \mathrm{~g} \mathrm{CAE} / \mathrm{kg}$ FW.

\section{Antioxidative activity}

In WEs, Okitanasu showed significantly high antioxidative activity similar to $1.0 \mathrm{mM}$ TL (Table 2). Activities of Mindennasu, Chijimina, and Kirariboshi were higher than those of $5.0 \mathrm{mM} \mathrm{AA}$ and $1.0 \mathrm{mM} \mathrm{BHT}$. Mosodake, Karatori stems, and Natsuna showed same activities as $0.1 \mathrm{mM}$ BHA, BHT, and TL, whereas, Fujisawakabu, Makomodake, and Tonojimakyuri were low. MEs from Kirariboshi and Chijimina possessed highest activities similar to $1.0 \mathrm{mM}$ TP and TL (Table 2). Natsuna, Mindennasu, and Okitanasu showed same activities as $5.0 \mathrm{mM}$ AA and $1.0 \mathrm{mM}$ BHA and BHT. Meanwhile, Makomodake was low. Ismail et al. (2004) measured antioxidant activities using $\beta$-carotene bleaching system and phenolic contents in cabbage, kale, shallots, spinach, and swamp cabbage. Shallots showed high activity about $69.1 \%$, followed by spinach, swamp cabbage, cabbage, and kale. Meanwhile, phenol content was highest in spinach, followed by in swamp cabbage, kale, shallots, and cabbage. Correlation was not observed between antioxidant activities and phenol contents.

\section{Superoxide anion radical scavenging activity}

In WEs, superoxide anion radical scavenging activity on Tomoefuki was highest about $90.4 \%$, followed by Makomodake, Natsuna, Atsumikabu, and Mindennasu, whereas Karatori stems were low about 32.3\% (Table 3). TESC was estimated to $0.11-0.33 \times 10^{3} \mathrm{mM}$ TE/ $\mathrm{kg} \mathrm{FW}$. In MEs, Tagawakabu possessed high activity about $88.7 \%$, 
Nagai, et al.

Table 2: Antioxidative activities of WEs and MEs from heirloom vegetables on Shonai region of Yamagata, Japan

\begin{tabular}{|c|c|c|c|c|c|c|}
\hline \multirow[t]{3}{*}{ Samples } & \multicolumn{6}{|c|}{ Absorbance $(500 \mathrm{~nm})$} \\
\hline & \multicolumn{2}{|c|}{$50 \mathrm{~min}$} & \multicolumn{2}{|c|}{$100 \mathrm{~min}$} & \multicolumn{2}{|c|}{$200 \mathrm{~min}$} \\
\hline & WEs & MEs & WEs & MEs & WEs & MEs \\
\hline Atsumikabu & $0.034 \pm 0.003$ & $0.006 \pm 0.001$ & $0.102 \pm 0.001$ & $0.062 \pm 0.003$ & $0.287 \pm 0.001$ & $0.069 \pm 0.004$ \\
\hline Tagawakabu & $0.057 \pm 0.001$ & $0.025 \pm 0.002$ & $0.128 \pm 0.002$ & 0.042 & $0.253 \pm 0.003$ & $0.163 \pm 0.004$ \\
\hline Fujisawakabu & $0.085 \pm 0.002$ & $0.008 \pm 0.002$ & 0.159 & $0.027 \pm 0.002$ & $0.389 \pm 0.006$ & $0.058 \pm 0.002$ \\
\hline Okitanasu & $0.013 \pm 0.006$ & $0.049 \pm 0.002$ & $0.028 \pm 0.009$ & $0.050 \pm 0.005$ & $0.037 \pm 0.008$ & $0.091 \pm 0.010$ \\
\hline Mindennasu & $0.039 \pm 0.005$ & $0.047 \pm 0.007$ & $0.049 \pm 0.014$ & $0.075 \pm 0.009$ & $0.056 \pm 0.003$ & $0.098 \pm 0.008$ \\
\hline Karatori (tubers) & 0 & $0.103 \pm 0.008$ & $0.141 \pm 0.005$ & $0.138 \pm 0.006$ & $0.189 \pm 0.007$ & $0.284 \pm 0.012$ \\
\hline Karatori (stems) & $0.056 \pm 0.003$ & $0.178 \pm 0.020$ & $0.105 \pm 0.008$ & $0.157 \pm 0.002$ & $0.148 \pm 0.004$ & $0.206 \pm 0.006$ \\
\hline Kirariboshi & 0.036 & $0.003 \pm 0.002$ & 0.031 & 0 & $0.064 \pm 0.003$ & $0.042 \pm 0.001$ \\
\hline Chijimina & $0.023 \pm 0.002$ & $0.014 \pm 0.002$ & 0.018 & 0 & $0.058 \pm 0.003$ & $0.043 \pm 0.002$ \\
\hline Natsuna & $0.019 \pm 0.002$ & $0.097 \pm 0.027$ & $0.015 \pm 0.003$ & $0.050 \pm 0.006$ & $0.168 \pm 0.001$ & $0.101 \pm 0.002$ \\
\hline Tonojimakyuri & $0.135 \pm 0.003$ & $0.116 \pm 0.013$ & $0.274 \pm 0.006$ & $0.189 \pm 0.008$ & $0.418 \pm 0.003$ & $0.286 \pm 0.011$ \\
\hline Tomoefuki & 0.040 & $0.029 \pm 0.003$ & 0.061 & $0.050 \pm 0.007$ & $0.250 \pm 0.002$ & $0.134 \pm 0.002$ \\
\hline Hirataakanegi & $0.152 \pm 0.005$ & $0.078 \pm 0.002$ & $0.261 \pm 0.014$ & $0.412 \pm 0.006$ & $0.367 \pm 0.011$ & $0.273 \pm 0.012$ \\
\hline Makomodake & $0.113 \pm 0.006$ & $0.103 \pm 0.021$ & $0.187 \pm 0.001$ & $0.196 \pm 0.001$ & $0.395 \pm 0.006$ & $0.429 \pm 0.003$ \\
\hline Mosodake & $0.040 \pm 0.001$ & 0.030 & 0.103 & 0.071 & $0.147 \pm 0.001$ & $0.148 \pm 0.003$ \\
\hline $1.0 \mathrm{mM} \mathrm{AA}$ & \multicolumn{2}{|c|}{$0.022 \pm 0.001$} & \multicolumn{2}{|c|}{$0.135 \pm 0.006$} & \multicolumn{2}{|c|}{$0.469 \pm 0.027$} \\
\hline $5.0 \mathrm{mM}$ AA & \multicolumn{2}{|c|}{$0.016 \pm 0.001$} & \multicolumn{2}{|c|}{$0.032 \pm 0.003$} & \multicolumn{2}{|c|}{$0.090 \pm 0.008$} \\
\hline $0.01 \mathrm{mM} \mathrm{BHA}$ & \multicolumn{2}{|c|}{$0.084 \pm 0.005$} & \multicolumn{2}{|c|}{$0.120 \pm 0.008$} & \multicolumn{2}{|c|}{$0.245 \pm 0.012$} \\
\hline $0.1 \mathrm{mM} \mathrm{BHA}$ & \multicolumn{2}{|c|}{$0.056 \pm 0.003$} & \multicolumn{2}{|c|}{$0.090 \pm 0.006$} & \multicolumn{2}{|c|}{$0.165 \pm 0.010$} \\
\hline $1.0 \mathrm{mM} \mathrm{BHA}$ & \multicolumn{2}{|c|}{$0.054 \pm 0.002$} & \multicolumn{2}{|c|}{$0.057 \pm 0.003$} & \multicolumn{2}{|c|}{$0.100 \pm 0.006$} \\
\hline $0.01 \mathrm{mM} \mathrm{BHT}$ & \multicolumn{2}{|c|}{$0.082 \pm 0.003$} & \multicolumn{2}{|c|}{$0.112 \pm 0.009$} & \multicolumn{2}{|c|}{$0.248 \pm 0.011$} \\
\hline $0.1 \mathrm{mM} \mathrm{BHT}$ & \multicolumn{2}{|c|}{$0.058 \pm 0.004$} & \multicolumn{2}{|c|}{$0.108 \pm 0.005$} & \multicolumn{2}{|c|}{$0.173 \pm 0.008$} \\
\hline $1.0 \mathrm{mM} \mathrm{BHT}$ & \multicolumn{2}{|c|}{$0.044 \pm 0.002$} & \multicolumn{2}{|c|}{$0.051 \pm 0.003$} & \multicolumn{2}{|c|}{$0.093 \pm 0.005$} \\
\hline $1.0 \mathrm{mM}$ TP & \multicolumn{2}{|c|}{0.006} & 0.0 & 001 & 0.0 & 002 \\
\hline $0.01 \mathrm{mM}$ TL & 0.0 & 005 & 0.0 & 006 & 0.2 & 013 \\
\hline $0.1 \mathrm{mM}$ TL & 0.0 & 002 & 0.0 & 003 & 0.1 & 008 \\
\hline $1.0 \mathrm{mM}$ TL & 0.0 & 001 & 0.0 & 002 & 0.0 & 002 \\
\hline Control & 0.3 & 008 & 0.7 & 025 & $1.4 \mathrm{C}$ & 041 \\
\hline
\end{tabular}

followed by Atsumikabu and Tomoefuki. Meanwhile, Karatori stems and tubers were low. TESC ranged from 0.04-0.32 x $10^{3} \mathrm{mM}$ TE $/ \mathrm{kg}$ FW. Correlation was not observed between activities on WEs or MEs and phenols or flavonoids contents. Kimura et al. (2002) reported that green tea possessed highest activity (197.2 U/g DW) among on 45 vegetables, while perilla was low about $20.0 \mathrm{U} / \mathrm{g}$ dry weight (U/g DW). Hirata (2010) investigated radical scavenging activities of 43 vegetables in Yamagata, Japan. High activity (IC50 $=0.0026 \mathrm{~g} / \mathrm{ml}$ extract $)$ was detected in eggplant (Tayanasu), whereas Chikuyo showed no activity. Onion (Yamaguchikodaka), bitter melon, and tomato (Momotaro) possessed high activities with IC50 $=0.004,0.0042$, and $0.005 \mathrm{~g} / \mathrm{ml}$ extract, respectively. Meanwhile, leaf mustards (Hikoshimaharuna, Miike) was low (IC50 $=0.0252 \mathrm{~g} / \mathrm{ml}$ extract). Turnip (Hagikoroge, Takehisa, and Suwan), bok choy, Chinese mustard, and rapeseed (Hanakkorr) did not detect the activities.

\section{Hydroxyl radical scavenging activity}

In WEs, Kirariboshi and Karatori stems showed remarkably high hydroxyl radical scavenging activities about 85.7 and $85.3 \%$, respectively, although activities did not reach that of $0.1 \mathrm{mM}$
TL (Table 3). Activity on Natsuna was similar to that in 0.01 mM BHT. On the contrary, Tonojimaky yri and Mindennasu were low. TESC ranged from 0.13-1.54 x $10^{3} \mathrm{mM}$ TE/ $\mathrm{kg}$ FW. In MEs, Tomoefuki exhibited significantly high activity about $88.2 \%$, followed by Karatori stems, whereas Mosodake was low about $34.3 \%$. TESC ranged from $0.39-1.59 \times 10^{3} \mathrm{mM}$ TE/ $\mathrm{kg}$ FW. Correlation was not observed between activities on WEs or MEs and phenols or flavonoids contents.

\section{DPPH radical scavenging activity}

In WEs, Tomoefuki and Kirariboshi possessed highest DPPH radical scavenging activities about 85.9 and $84.5 \%$, respectively, whereas Makomodake and Mosodake were low (Table 3). Particularly, Tonojimakyuri was lowest. TESC ranged from 26.4-150.6 × $10^{3} \mathrm{mM}$ TE/kg FW. In MEs, activities of Tagawakabu and Fujisawakabu were significantly high (86.6 and 86.3\%, respectively). Tonojimakyuri was lowest among these vegetables. TESC ranged from 23.9-152.0 x $10^{3} \mathrm{mM} \mathrm{TE} / \mathrm{kg}$ FW. DPPH radical scavenging activity is correlated with phenol contents (Velioglu et al., 1998). However, correlation was not observed between activities of WEs or MEs and phenols or flavonoids contents. Yamaguchi et al. (2012) investigated DPPH radical 
Table 3: Superoxide anion radicals, hydroxyl radicals, and DPPH radicals scavenging activities of WEs and MEs from heirloom vegetables on Shonai region of Yamagata, Japan

\begin{tabular}{|c|c|c|c|c|c|c|}
\hline \multirow[t]{3}{*}{ Samples } & \multicolumn{6}{|c|}{ Scavenging activity (\%) } \\
\hline & \multicolumn{2}{|c|}{ Superoxide anion radicals } & \multicolumn{2}{|c|}{ Hydroxyl radicals } & \multicolumn{2}{|c|}{ DPPH radicals } \\
\hline & WEs & MEs & WEs & MEs & WEs & MEs \\
\hline Atsumikabu & $82.3 \pm 3.9(0.30)$ & $76.0 \pm 2.1(0.27)$ & $46.2 \pm 5.3(0.66)$ & $52.9 \pm 5.8(0.80)$ & $76.3 \pm 1.5(130.8)$ & $78.8 \pm 0.9(136.0)$ \\
\hline Tagawakabu & $71.6 \pm 4.2(0.26)$ & $88.7 \pm 3.0(0.32)$ & $47.8 \pm 4.9(0.69)$ & $76.5 \pm 5.9(1.33)$ & $72.6 \pm 1.7(123.2)$ & $86.6 \pm 1.1(152.0)$ \\
\hline Fujisawakabu & $77.8 \pm 3.5(0.28)$ & $74.1 \pm 5.2(0.27)$ & $52.9 \pm 6.7(0.80)$ & $54.4 \pm 6.0(0.84)$ & $74.7 \pm 2.0(127.6)$ & $86.3 \pm 1.9(151.5)$ \\
\hline Okitanasu & $65.6 \pm 4.2(0.23)$ & $60.8 \pm 1.9(0.22)$ & $62.2 \pm 6.4(1.01)$ & $73.3 \pm 4.7(1.26)$ & $82.9 \pm 1.1(144.4)$ & $79.9 \pm 1.5(138.3)$ \\
\hline Mindennasu & $81.9 \pm 3.8(0.29)$ & $57.5 \pm 5.7(0.20)$ & $24.4 \pm 4.1(0.17)$ & $74.2 \pm 6.0(1.28)$ & $28.8 \pm 2.9(33.0)$ & $60.7 \pm 2.2(98.7)$ \\
\hline Karatori (tubers) & $50.2 \pm 3.3(0.18)$ & $12.9 \pm 5.1(0.04)$ & $61.0 \pm 8.2(0.99)$ & $62.1 \pm 4.5(1.01)$ & $70.1 \pm 1.5(118.1)$ & $76.1 \pm 1.8(130.4)$ \\
\hline Karatori (stems) & $32.3 \pm 6.9(0.11)$ & $10.7 \pm 2.8(0.03)$ & $85.3 \pm 3.9(1.53)$ & $81.3 \pm 2.1(1.44)$ & $55.1 \pm 1.4(87.2)$ & $55.6 \pm 2.4(88.2)$ \\
\hline Kirariboshi & $70.0 \pm 4.7(0.25)$ & $33.8 \pm 14.1(0.11)$ & $85.7 \pm 2.8(1.54)$ & $73.5 \pm 2.8(1.27)$ & $84.5 \pm 1.8(147.7)$ & $83.5 \pm 1.9(145.7)$ \\
\hline Chijimina & $60.7 \pm 5.4(0.22)$ & $41.2 \pm 10.2(0.14)$ & $74.8 \pm 8.8(1.29)$ & $70.9 \pm 3.4(1.21)$ & $73.7 \pm 2.7(125.5)$ & $78.2 \pm 2.0(134.8)$ \\
\hline Natsuna & $83.4 \pm 8.5(0.30)$ & $48.5 \pm 12.1(0.17)$ & $82.1 \pm 6.9(1.46)$ & $66.3 \pm 4.6(1.10)$ & $80.3 \pm 1.1(139.1)$ & $79.4 \pm 2.3(137.2)$ \\
\hline Tonojimakyuri & $64.7 \pm 3.8(0.23)$ & $38.5 \pm 4.9(0.13)$ & $22.7 \pm 3.3(0.13)$ & $53.2 \pm 7.1(0.81)$ & $10.3 \pm 2.7(n d)$ & $12.8 \pm 2.7(n d)$ \\
\hline Tomoefuki & $90.4 \pm 2.1(0.33)$ & $74.6 \pm 2.1(0.27)$ & $42.2 \pm 5.0(0.57)$ & $88.2 \pm 4.2(1.59)$ & $85.9 \pm 1.2(150.6)$ & $68.9 \pm 1.8(115.6)$ \\
\hline Hirataakanegi & $56.0 \pm 4.5(0.20)$ & $37.0 \pm 4.3(0.13)$ & $78.8 \pm 7.8(1.38)$ & $65.6 \pm 5.8(1.09)$ & $80.0 \pm 0.9(138.5)$ & $76.4 \pm 2.0(131.1)$ \\
\hline Makomodake & $85.8 \pm 5.2(0.31)$ & $68.8 \pm 3.6(0.25)$ & $75.1 \pm 4.9(1.30)$ & $72.9 \pm 6.1(1.25)$ & $25.6 \pm 2.8(26.4)$ & $23.9 \pm 2.5(22.9)$ \\
\hline Mosodake & $70.5 \pm 4.6(0.25)$ & $53.7 \pm 3.5(0.19)$ & $72.1 \pm 9.7(1.23)$ & $34.3 \pm 5.9(0.39)$ & $28.8 \pm 4.1(33.0)$ & $29.3 \pm 2.8(34.0)$ \\
\hline $1.0 \mathrm{mM} \mathrm{AA}$ & \multicolumn{2}{|c|}{$14.7 \pm 0.2$} & \multicolumn{2}{|c|}{$13.2 \pm 0.2$} & \multicolumn{2}{|c|}{$3.1^{*}$} \\
\hline $5.0 \mathrm{mM} \mathrm{AA}$ & \multicolumn{2}{|c|}{$89.9 \pm 5.3$} & \multicolumn{2}{|c|}{$17.6 \pm 0.7$} & \multicolumn{2}{|c|}{$34.1 \pm 2.0^{* *}$} \\
\hline $0.01 \mathrm{mM} B \mathrm{BH}$ & \multicolumn{2}{|c|}{$29.3 \pm 0.5$} & \multicolumn{2}{|c|}{$59.1 \pm 0.8$} & \multicolumn{2}{|c|}{5.5} \\
\hline $0.1 \mathrm{mM} \mathrm{BHA}$ & \multicolumn{2}{|c|}{$36.4 \pm 0.9$} & \multicolumn{2}{|c|}{$93.3 \pm 1.4$} & \multicolumn{2}{|c|}{$17.5 \pm 0.4$} \\
\hline $1.0 \mathrm{mM} \mathrm{BHA}$ & \multicolumn{2}{|c|}{$51.9 \pm 1.4$} & \multicolumn{2}{|c|}{$95.2 \pm 1.4$} & \multicolumn{2}{|c|}{$72.7 \pm 3.6$} \\
\hline $0.01 \mathrm{mM} \mathrm{BHT}$ & \multicolumn{2}{|c|}{$11.7 \pm 0.2$} & \multicolumn{2}{|c|}{$82.8 \pm 0.9$} & \multicolumn{2}{|c|}{3.9} \\
\hline $0.1 \mathrm{mM} \mathrm{BHT}$ & \multicolumn{2}{|c|}{$46.6 \pm 1.0$} & \multicolumn{2}{|c|}{$97.6 \pm 1.6$} & \multicolumn{2}{|c|}{$7.9 \pm 0.1$} \\
\hline $1.0 \mathrm{mM} \mathrm{BHT}$ & \multicolumn{2}{|c|}{$48.4 \pm 1.2$} & \multicolumn{2}{|c|}{$>100$} & \multicolumn{2}{|c|}{$31.7 \pm 0.8$} \\
\hline $1.0 \mathrm{mM}$ TP & \multicolumn{2}{|c|}{$52.6 \pm 4.2$} & \multicolumn{2}{|c|}{$67.6 \pm 4.3$} & & 2.8 \\
\hline $0.01 \mathrm{mM} \mathrm{TL}$ & 46 & \pm 1.0 & 81. & 0.6 & & \\
\hline $0.1 \mathrm{mM}$ TL & 58 & \pm 1.1 & 91. & 1.2 & & 0.2 \\
\hline $1.0 \mathrm{mM}$ TL & 76 & \pm 1.9 & & & 86 & 3.3 \\
\hline
\end{tabular}

${ }^{*} 0.1 \mathrm{mM} \mathrm{AA} ;{ }^{* *} 1.0 \mathrm{mM}$ AA. Values in brackets are $10^{3}$ millimoles of TL equivalents per $\mathrm{kg}$ of fresh weight of vegetables.

scavenging activities of Yamatoyasai vegetables. Kaorigobo showed highest TESC about $37.9 \times 10^{3} \mathrm{mM}$ TE/kg FW, followed by Udakingobo and Koshoga, whereas Hanshirokyuri and Yamatosanzyakukyuri exhibited low TESC (0.35 and $0.34 \times 10^{3} \mathrm{mM}$ TE/kg FW, respectively). Correlation was shown with $\mathrm{R}^{2}=0.745$ between oxygen radical absorbance capacity and polyphenol contents. Itou et al. (2010) reported that lotus root possessed highest TESC $(7.6 \mathrm{mM}$ TE/kg FW), followed by Shodaikon (1.9 mM TE/kg FW), whereas elephant foot (tubers) was lowest $(0.4 \mathrm{mM}$ TE/ $\mathrm{kg} \mathrm{FW})$ on local agricultural products in Ehime, Japan. Pea (Orandaendo) showed highest TESC about $4.7 \mathrm{mM}$ TE/kg FW, while loquat hacha was lowest. Meanwhile, common henbit (20.8 $\mathrm{mM} \mathrm{TE} / \mathrm{kg} \mathrm{FW}$ ) and Jersey cudweed (Gogyo) (15.8 mM TE/kg FW) possessed significantly higher activities than other vegetables. Correlation was not observed between TESC and polyphenol contents. Hirata (2010) reported DPPH radical scavenging activities of agricultural products in Yamaguchi, Japan. TESC was highest in crown daisy (Oba) (9.0 mM TE/kg FW) and burdock (Takinokawa) (8.7 $\mathrm{mM}$ TE/kg FW), but few activities were detected on leaf mustard, bok choy, and Chinese mustard. Kujonegi showed middle activity $(2.9 \mathrm{mM}$ TE/ $\mathrm{kg} \mathrm{FW})$.

\section{ACE inhibitory activity}

WEs on all vegetables showed high ACE inhibitory activities (Table 4). Atsumikabu, Tagawakabu, Okitanasu, and Makomodake possessed high activities of more than 90\%, followed by Mosodake, Tonojimakyuri, Karatori stems, and Mindennasu. Natsuna was middle activity about $52.7 \%$. While, every ME showed high activity of more than $71.1 \%$ except for Tomoefuki. Particularly, Fujisawakabu, Okitanasu, Tonojimakyuri, and Mosodake exhibited significantly high activities about 93.1-95.8\%. Correlation with $\mathrm{R}^{2}=0.621$ was observed between activities on MEs and flavonoids contents. Nicotianamine is an ACE inhibitor in mushrooms (Izawa and Aoyagi, 2006). Correlation was shown between its contents on various beans and these activities (Izawa et al., 2008). Izawa and Aoyagi (2012) measured ACE inhibitory activities and nicotianamine contents in 80 vegetables. Surprisingly, asparagus and hosta (Urui) showed high activities, although these did not contain nicotianamine. Kimura et al. (2007) investigated ACE inhibitory activities on 7 heirloom vegetables in Yamanashi, Japan. Mizukakena (mizuna) showed high activity (IC50 = $8.8 \mathrm{~g} / 100 \mathrm{ml}$ extract), followed by Mizukakena (fuyuna). Enomoto (2003) report that Nakajimana and 
Nagai, et al.

Table 4: ACE and hyaluronidase inhibitory activities of WEs and MEs of heirloom vegetables on Shonai region of Yamagata, Japan

\begin{tabular}{lcccr}
\hline \multirow{2}{*}{ Vegetables } & \multicolumn{2}{c}{ ACE inhibitory activity $(\%)$} & & \multicolumn{2}{c}{ Hyaluronidase inhibitory activity $(\%)$} \\
\cline { 2 - 3 } & WEs & MEs & WEs & MEs \\
\hline Atsumikabu & $90.6 \pm 4.1$ & $82.8 \pm 3.5$ & $83.8 \pm 3.9(1.43)$ & $43.3 \pm 2.1(0.80)$ \\
Tagawakabu & $94.5 \pm 4.5$ & $89.4 \pm 4.7$ & $60.1 \pm 4.1(1.07)$ & $79.1 \pm 1.2(1.35)$ \\
Fujisawakabu & $65.5 \pm 4.7$ & $93.3 \pm 3.8$ & $76.5 \pm 3.2(1.32)$ & $48.1 \pm 5.9(0.87)$ \\
Okitanasu & $95.3 \pm 4.2$ & $95.8 \pm 5.0$ & $18.9 \pm 5.1(0.42)$ & $88.1 \pm 3.7(1.50)$ \\
Mindennasu & $84.5 \pm 3.8$ & $72.9 \pm 1.6$ & $40.4 \pm 1.9(0.75)$ & $51.5 \pm 4.1(0.93)$ \\
Karatori (tubers) & $72.9 \pm 8.5$ & $82.3 \pm 3.7$ & $65.5 \pm 3.0(1.14)$ & $87.4 \pm 0.8(1.49)$ \\
Karatori (stems) & $86.3 \pm 4.3$ & $81.8 \pm 3.2$ & $74.5 \pm 1.5(1.29)$ & $92.7 \pm 1.0(1.58)$ \\
Kirariboshi & $75.6 \pm 4.0$ & $71.1 \pm 5.9$ & $38.9 \pm 6.1(0.72)$ & $84.2 \pm 0.6(1.44)$ \\
Chijimina & $62.1 \pm 18.2$ & $76.1 \pm 5.1$ & $52.4 \pm 4.0(0.95)$ & $50.1 \pm 1.2(0.90)$ \\
Natsuna & $52.7 \pm 6.5$ & $80.6 \pm 4.2$ & $4.7 \pm 2.5(0.20)$ & $31.9 \pm 6.0(0.62)$ \\
Tonojimakyuri & $86.6 \pm 5.7$ & $93.1 \pm 4.4$ & $32.7 \pm 1.2(0.63)$ & $6.2 \pm 3.9(0.21)$ \\
Tomoefuki & $69.6 \pm 10.1$ & $45.1 \pm 8.2$ & $38.6 \pm 4.9(0.72)$ & $39.4 \pm 6.9(0.74)$ \\
Hirataakanegi & $81.2 \pm 3.8$ & $83.0 \pm 3.6$ & $14.0 \pm 3.8(0.33)$ & $37.9 \pm 1.5(0.72)$ \\
Makomodake & $90.6 \pm 6.5$ & $87.4 \pm 2.5$ & $16.0 \pm 3.5(0.38)$ & $57.6 \pm 2.6(1.02)$ \\
Mosodake & $89.0 \pm 4.9$ & $95.7 \pm 4.3$ & $28.0 \pm 5.4(0.56)$ \\
\hline Valus
\end{tabular}

Values in brackets are $10^{2}$ millimoles of sodium cromoglicate equivalents per $\mathrm{kg}$ of fresh weight of vegetables.

Futatsukakarashina possessed strongly activities among 12 heirloom vegetables in Ishikawa, Japan.

\section{Hyaluronidase inhibitory activity}

Sodium cromoglicate (SC) is used as one of commercially available anti-allergic drug against atopic dermatitis based on food allergies, allergic rhinitis, and allergic conjunctivitis. Hyaluronidase inhibitory activities were also expressed as millimoles of SC equivalents per $\mathrm{kg}$ of fresh weight of vegetables [SC equivalents inhibitory capacity (SCEIC); $\mathrm{mM}$ SCE $/ \mathrm{kg}$ FW]. In WEs, Atsumikabu and Karatori stems showed extremely high activities about 83.3 and $81.7 \%$, respectively, followed by Fujisawakabu and Kirariboshi (Table 4). Meanwhile, Okitanasu, Makomodake, and Mosodake were low about 14.0-18.9\%. SCEIC was estimated to 0.20-1.43 x $10^{2} \mathrm{mM}$ SCE/ kg FW. In MEs, Karatori stems possessed significantly high activity about $92.7 \%$, followed by Okitanasu, Karatori tubers, and Kirariboshi. Fujisawakabu, Mindennasu, Chijimina, and Makomodake showed middle activities ranging from 48.1-57.6\%, whereas Tonojimakyuri was lowest. SCEIC ranged from $0.21-1.58 \times 10^{2} \mathrm{mM} \mathrm{SCE/}$ $\mathrm{kg}$ FW. Correlation was not observed between activities and phenols or flavonoids contents. Ippoushi et al. (2000) investigated hyaluronidase inhibitory activities on 46 vegetables and herb extracts. Extracts prepared from 8 Labiatae plants and borage showed activities. Moreover, they isolated and identified rosmarinic acid as inhibitor from lemon balm extract. Kimura et al. (2007) reported that activities on 7 heirloom vegetables in Yamanashi, Japan were low or not detected. Hirata (2010) elucidated that none of activities were low, although 32 vegetables exhibited activities among 43 vegetables in Yamaguchi, Japan.

In this study, it was revealed for the first time the nutritional and health-promoting properties of representative heirloom vegetables on Shonai region of Yamagata, Japan. Further research is going to investigate the properties of other vegetables because of existence of many native varieties of vegetables in this area. It is also necessary to research the nutrients and functionalities on each part of vegetable. Cooking may affect the contents of useful components in vegetables. Detailed analysis of nutritional and functional properties on vegetables before and after cooking is in progress to develop novel processing methods of these vegetables.

\section{CONCLUSIONS}

These findings demonstrated that heirloom vegetables used in this study served as good sources of vitamins, phenolics, and antioxidants compared to corresponding commercially available vegetables. Positively eating of these vegetables can probably contribute to health promotion to prevent life style-related diseases such as cancer, hypertension, and inflammation. Furthermore, it also may have potentials for preservation of species and for promotion of sustainable cultivation of heirloom vegetables.

\section{ACKNOWLEDGEMENT}

This work was financially supported in part by a grant from the Egashira Foundation, Japan.

\section{CONFLICT OF INTEREST}

Authors have declared that no competing interests exist.

\section{Author contributions}

All authors designed the work, acquired, analyzed, interpreted the data, and wrote and revised the manuscript. 


\section{REFERENCES}

Association to Protect Traditional Vegetables in Aizu. 2010. AizuNousho. Available from: http://www.aizu-city.net/nousyo/d-yasai. htm. [Last retrieved on 2018 Oct 03].

Enomoto, T. 2003. Nutrition of agricultural and marine products in Hokuriku distinct and its utilization for processing foods. Nippon Shokuhin Kagaku Kogaku Kaishi. 50: 379-385.

Fawole, O. A., N. P. Makunga and U. L. Opara. 2012. Antibacterial, antioxidant and tyrosinase-inhibition activities of pomegranate fruit peel methanolic extract. BMC Complement. Altern. Med. 12: $200-218$.

Fidrianny, I., N. A. S. Anggraeni and M. Insanu. 2018. Antioxidant properties of peels extracts from three varieties of banana (Musa sp.) grown in West Java-Indonesis. Int. Food Res. J. 25: 57-64.

Hirata, T. 2010. Anti-oxidant, anti-allergic and lipid metabolic improvement function of agricultural products in Yamaguchi prefecture. Bull. Yamaguchi Prefectural Technol. Cent. Agric. For. 1: 1-10.

Ippoushi, K., Y. Yamaguchi, H. Itou, K. Azuma and H. Higashio. 2000. Evaluation of inhibitory effects of vegetables and herbs on hyaluronidase and identification of rosmaric acid as a hyaluronidase inhibitor in lemon balm (Melissa officinalis L.). Food Sci. Technol. Res. 6: 74-77.

Ismail, A., Z. M. Marjan and C. W. Foong. 2004. Total antioxidant activity and phenolic content in selected vegetables. Food Chem. 87: 581-586.

Isobe, Y., H. Kikuzaki, S. Kubo, Y. Tachibana, S. Doke, M. Honzawa and S. Mizuno. 2011. Calculation of energy value. In: Fujita, S. and Yamada, K. (Eds.), Experimental Book in Food Science. 2nd ed. Ishiyaku Publishers, Tokyo, Japan, p. 43.

Itou, F., S. Minakuchi and H. Ishiishikawa. 2010. Evaluation of antioxidant activity and polyphenol content of local agricultural products in Ehime Prefecture. Bull. Ehime Res. Inst. Agric. For. Fish. 2: 43-51.

Izawa, H. and Y. Aoyagi. 2006. Inhibition of angiotensin converting enzyme by mushroom. Nippon Shokuhin Kagaku Kogaku Kaishi. 53: 459-465.

Izawa, H. and Y. Aoyagi. 2012. Nicotianamine contents among vegetables and their inhibitory activity of angiotensin-I converting enzyme. Nippon Shokuhin Kagaku Kogaku Kaishi. 59: 348-353.

Izawa, H., N. Yoshida, N. Shiragai and Y. Aoyagi. 2008. Nicotianamine content in various beans and its inhibition activity of angiotensin-I converting enzyme. Nippon Shokuhin Kagaku Kogaku Kaishi. 55: 253-257.

JA-Kyoto. 2007. Kyo-Vegetables. Available from: https://www.jakyoto. com/kyoyasai. [Last retrieved on 2018 Oct 03].

Kagawa, A. 2018. Standard Tables of Food Composition in Japan 2018. Kagawa Nutrition University Publishing Division, Tokyo, Japan.

Kanazawa City Agricultural Products Branding Association. 2009. Kaga-Vegetables. Available from: http://www.kanazawakagayasai.com. [Last retrieved on 2018 Oct 03].

Kim, D. O., O. K. Chun and Y. J. Kim. 2003. Quantification of polyphenolics and their antioxidant capacity in fresh plums. J.
Agric. Food Chem. 51: 6509-6515.

Kimura, H., K. Naganuma, M. Kojima and T. Onda. 2007. ACE inhibiting activity and the antiallergic activity of local agricultural products. Y CROST Res. Rep. 2: 1-5.

Kimura, T., K. Yamagishi, M. Suzuki and H. Shinmoto. 2002. Relative estimation of the radical scavenging activities of agricultural products. Nippon Shokuhin Kagaku Kogaku Kaishi. 49: 257-266.

Miura, Y., Y. Fukunaga, Y. Takigawa, M. Tsuda, Y. Watanabe and I. Seyama. 2006. Determination of acidity and alkalinity of food materials. Bull. Hiroshima Jogakuin Univ. 56: 121-129.

Mizuno, T. and T. Sumino. 2008. Functional components of traditional vegetables in Aizu region. J. Cookery Sci. Jpn. 41: 164-165.

Nagai, T., N. Kai, Y. Tanoue and N. Suzuki. 2018. Chemical properties of commercially available honey species and the functional properties of caramelization and Maillard reaction products derived from these honey species. J. Food Sci. Technol. 55: 586-597.

Nagata, M. and I. Yamashita. 1992. Simple method for simultaneous determination of chlorophyll and carotenoids in tomato fruit. Nippon Shokuhin Kogyo Gakkaishi. 39: 925-928.

Nakamura, K., Takita, T., Watanabe, T., Sato, H., and Wada, M. (1998). Vitamin. In: Nakamura, K., T. Takita and T. Watanabe, (Eds.), Experimental Book in Basic Food Science. Sankyo Shuppan, Tokyo, Japan, pp. 137-144.

Ross, J. A. and C. M. Kasum. 2002. Dietary flavonoids: Bioavailability, metabolic effects, and safety. Annu. Rev. Nutr. 22: 19-34.

Slinkard, K. and V. L. Singleton. 1977. Total phenol analysis: Automation and comparison with manual methods. Am. J. Enol. Vitic. 28: 49-55

Sugawara, K. and M. Soejima. 1996. Experimental Methods of Biochemistry. Gakkai Shuppan Center, Tokyo, Japan, pp. 182-183.

The Vitamin Society of Japan. 1990. Vitamin Handbook. Kagakudojin, Kyoto, Japan, pp. 139-140.

Tomiyoshi, M. and S. Ueno. 2016. Characteristics of perception and consumption of traditional vegetables by local residents and tourists: Case study of Kumamoto prefecture. J. Rural Probl. 52: 229-234.

Tsuruoka Creative City of Gastronomy Promotion Committee. 2018. To Become a "Utopia of Food". Available from: http://www. creative-tsuruoka.jp/news-info/zairaisakumotu-tsuruoka2018. html. [Last retrieved on 2018 Oct 03].

Velioglu, Y. S., G. Mazza, L. Gao and B. D. Oomah. 1998. Antioxidant activity and total phenolics in selected fruits, vegetables, and grain products. J. Agric. Food Chem. 46: 4113-4117.

Verma, M., G. K. Rai and D. Kaur. 2018. Effect of extraction solvents on phenolic content and antioxidant activities of Indian gooseberry and guava. Int. Food Res. J. 25: 762-768.

Yamagata Forum for the Indigenous Crops. 2012. Indigenous Crops in Yamagata. 3rd ed. Yamagata University Press, Yamagata, Japan.

Yamaguchi, T., H. Hara, T. Nishimoto, T. Matoba and H. Takamura. 2012. Evaluation of the proximate components and antioxidant activity of yamato yasai, the traditional vegetables of Nara. J. Cookery Sci. Jpn. 45: 197-203. 